\section{AB1023 ENHANCED RENAL TRANSPORTER ACTIVITIES OF OAT1 AND OAT3 BY KEISHIBUKURYOGAN (K-06) AND IN VIVO URIC ACID MODULATING EFFECT AT POTASSIUM OXONATE-INDUCED MOUSE SETTING}

D.-S. Oh ${ }^{1}$, H.J. Shin ${ }^{2,3}$, S.H. Lee ${ }^{1}$, S.-M. Oh ${ }^{1}{ }^{1}$ The K-herb Research Centre, Korea Institute of Oriental Medicine, Daejeon; ${ }^{2}$ Department of Pharmacology and Pharmaco Genomics Research Centre, College of Medicine, Inje Univ, ${ }^{3} D M P K$, SPMED, Busan, Korea, Republic of Ireland

Background: Studies on renal solute carrier transporters have made the renal pathophysiology be in progress towards more mechanistic and the knowledge on renal drugs including drug-drug interaction be more evidentiary. Among the evidence, uricosuric phenomenon has been known that rodent experimental model is also useful for predicting human uricosuria.

Objectives: The aim of this study was to assess the substrate uptake at the overexpression of renal transporters, OAT1, OAT3 and URAT1 at kidney proximal tubular cell lines with or without a commercial herbal medicine, Keishibukuryogan (K06) and to further measure serum and urinary uric acid concentrations in the setting of potassium oxonate (PO)-induced ICR mouse model with or without K-06. Methods: The transporter-expressed HEK293-OAT1 and HEK293-OAT3 cells were seeded on BD poly-d-lysine microplates to uptake the $\left[{ }^{3} \mathrm{H}\right]$ estrone sulfate for $5 \mathrm{~min}$ in absence or presence of K-06. URAT1 was overexpressed using Xenopus oocytes being injected with in vitro-copied RNA of URAT1, and then to measure the uptake of $\left[{ }^{14} \mathrm{C}\right]$ uric acid with/without $\mathrm{K}-06$. Total radioactivity was measured using a liquid scintillation counter. Serum and urinary uric acid was measured in PO ICR mice after three-day intake of K-06. They were assigned by 4 per each group; 1) control group, 2) PO-induced group, 3) PO-induced with allopurinol $50 \mathrm{mg} / \mathrm{kg} /$ day intake group and 4) PO-induced with allopurinol plus K-06 $300 \mathrm{mg} / \mathrm{kg} / \mathrm{day}$ intake group.

Results: To determine the kinetic parameters of concentration-dependent uptake of overexpressed OAT1 and OAT3 transporters in HEK293 cells, the K-06 inhibitory parameters on OAT1 and OAT3 were presented with the $\mathrm{IC}_{50}$ values of 49.3 and $31.5 \mu \mathrm{g} / \mathrm{mL}$, respectively. The K-06 inhibited URAT1 with $\mathrm{IC}_{50}$ of $59.3 \mu \mathrm{g} / \mathrm{mL}$. The K-06 $(300 \mathrm{mg} / \mathrm{kg})$ reduced serum levels of uric acid approximately $30 \% \mathrm{com}$ pared to that of PO-control group $(p=0.039)$ and K-06 showed the slight elevation of urinary uric acid by $12 \%$ compared to that of $\mathrm{PO}$-control group with no statistical significance.

Conclusions: The present findings demonstrated that the K-06 modulated basolateral and apical renal transporters and the K-06 showed the slight increased uric acid excretion and the uric acid lowering effect in experimental mouse setting.

\section{REFERENCES:}

[1] Becker GJ, Hewitson TD. Animal models of chronic kidney disease: useful but not perfect. Nephrol Dial Transplant 2013;28:2432-8. doi: 10.1093/ndt/ gft071

[2] Key difference between mouse and human kidney cells. Which is available at https://www.sciencedaily.com/releases/2016/03/160318181600.htm

Acknowledgements: This study was supported by the Traditional Korean Medicine $R$ and $D$ program funded by the Ministry of Health and Welfare through the Korea Health Industry Development Institute (KHIDI, Grant \# HI16C0864). The commercial herbal products, Keishibukuryogan (K-06) were donated by the virtue of Kracie Pharma, Co., Ltd.

Disclosure of Interest: None declared

DOI: 10.1136/annrheumdis-2018-eular.4369

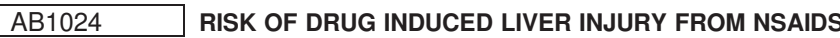 IN PATIENTS WITH GOUT}

E. Mikhnevich ${ }^{1}$, T. Pavlovich ${ }^{1}$, T. Rayeuneva $^{1}$, E. Mytnik ${ }^{2}$, E. Leonchik ${ }^{3}$, V. Tettegah ${ }^{4} .{ }^{1}$ Department Of Internal Medicine, UNIVERSITY OF MEDICINE: ${ }^{2}$ Department Of Rheumatology, Diagnostic Center, ${ }^{3}$ Emergency Department, Hospital \#5; ${ }^{4}$ UNIVERSITY OF MEDICINE, Minsk, Belarus

Background: Drug Induced Liver Injury (DILI) in patients under nonsteroidal antiinflammatory drugs (NSAIDs) is more frequently presented by hepatocellular damage when serum alanine aminotransferase (ALT) level exceeds 2 times (or more) the upper limits of norms. ${ }^{1}$ The value of minimal hypertransaminasemia (MHTE) (when serum ALT exceeds the upper limit of normal up to twice) is still not clear today.

Objectives: To define the terms of DILI development and the value of MHTE in patients with gouty arthritis (GA) under NSAIDs.

Methods: 189 patients $(25,6 \%)$ of 738 with $\mathrm{GA}^{\mathrm{ACR}, 1977}$ were included in our retrospective study. At the onset of gout attack, all patients had normal basal serum ALT, which elevated after starting NSAIDs therapy. Pre-existing liver impairment was not registered. Patients were divided into 2 groups according to the changes in serum ALT after NSAIDs treatment: patients with MHTE $(n=101)$ and those with
DILI $(n=88)$. The mean age of $55^{49-60}$ years and $54^{44-59,5}$ years as well as the sex distribution (men $90,1 \%$ and $93,3 \%$, respectively) were compatible in both the groups $(p<0,05)$. In DILI group, elevated serum ALT exceeded $2-3$ times the upper norms in $81,8 \%(n=72)$ of patients; $3-5$ times in $14,8 \%(n=5)$; more than 5 times in $3,4 \%(n=3)$ with the mean serum ALT being of $89^{75-108,5} \mathrm{U} / \mathrm{L}$. In MHTE group, serum ALT exceeded the upper norms by more than $50 \%$ in $50,5 \%(n=51)$ of cases. After NSAIDs therapy, the mean serum ALT of $51\left({ }^{47-57} \mathrm{U} / \mathrm{L}\right.$ was revealed.

Results: The mean duration of NSAIDs therapy in the groups made $8^{5-10}$ days in MHTE group and $10^{6-14}$ days in DILI group. Statistically significant difference $(U=3236, p<0,001)$ was revealed between the groups when comparing the duration of NSAIDs treatment. In addition, in DILI group, $97,7 \%$ of patients received NSAIDs in high doses $\left(\chi^{2}=19,4 ; p<0,001\right)$ during all period of treatment. ROC analysis showed that the probability of DILI development decreased in patients with GA taking NSAIDs in high doses during less than 11 days ( $A \cup C=0,64 \pm 0,04$, $\mathrm{p}=0,010, \mathrm{~S}=47,7 \%, \mathrm{Sp}-82,2 \%, \mathrm{OR}-4,21,95 \% \mathrm{Cl}-3,38-5,24)$.

Conclusions: $25,6 \%$ of patients with GA have demonstrated elevated serum ALT after NSAIDs therapy: MHTE developed in $13,7 \%$ of cases, DILI in $11,9 \%$. Our study showed that MHTE developed in the period less than 11 days of high dose NSAIDs treatment with DILI being likely to occur during more prolonged treatment. MHTE group may be considered as a risk group for DILI development.

\section{REFERENCE:}

[1] Aithal G. P. Hepatotoxicity related to antirheumatic drugs. Nat. Rev. Rheumatol. 2011; 7: 139-150.

Disclosure of Interest: None declared

DOI: 10.1136/annrheumdis-2018-eular.3130

\section{AB1025 MONOSODIUM URATE CRYSTAL FORMATIONS FROM TOPHI IN SYNOVIAL FLUID}

E. Pascual ${ }^{1}$, M. Andrés ${ }^{2,3}$, F. Sivera ${ }^{4} .{ }^{1}$ Emeritus Professor, Universidad Miguel Hernández; ${ }^{2}$ Seccion de Reumatologia, Hospital General Universitario De Alicante-Isabial; ${ }^{3}$ Dpt Medicina Clínica, Universidad Miguel Hernández; ${ }^{4}$ Seccion De Reumatologia, Hospital General Universitario de Elda, Alicante, Spain

Background: At the joints MSU crystals form primarily at the cartilage surface; on occasions also tophi form at joint margins. Most often monosodium urate (MSU) crystals at the synovial fluid (SF) are found isolated. In tophi MSU crystals often show as spherulitic formations, fanning away from a central point ${ }^{1}$ (Figure 1 200x, polarised light). We have detected in synovial fluid formations of MSU crystals with an organisation indicative that they formed in tophi, draining later to the fluid. The formations also help to understand how crystals formed in tophi. Methods: Our photographic archive of SF have been reviewed.

Results: Two types of formations are found. A) A spherulitic formation where the crystals fan radially as in the segment of a sphere (Figure, middle, 600x, polarised light). B) Paired crystals bound longitudinally to each other (Figure, right, 600x phase contrast).

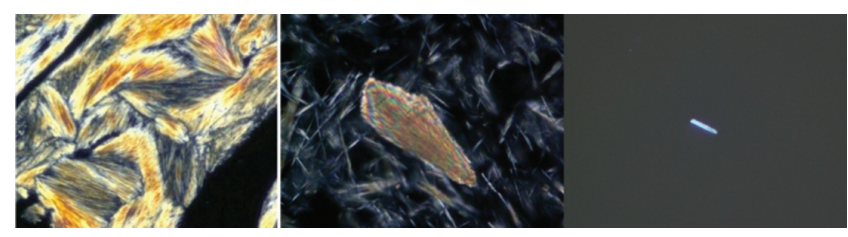

Abstract AB1025 - Figure 1

Conclusions: At tophi spherulitic crystal formations are usual (figure 1) in which MSU crystals radiate as in a fan. A) Pieces of these same formations, seen as the segment of a sphere, are occasionally seen in SF (Figure 2), usually containing a large number of crystals and suggesting that they have drained from a tophus. Likely to build these formations, the initial crystals served as a template on which successive crystals formed by epitaxia, - the crystal formation method of least energy requirement -, explaining the rapid growth that tophi can present. Their unimpeded migration to the joint cavity suggest that they formed freely and unconnected to any organic structure within the tophus. B) In SF containing large numbers of crystals, paired crystals - two crystals lying side by side and usually of similar length and width - are also found. Their paired position likely indicates that one served as template to the other, or that they grew together sharing a crystal net - twin crystals. In all, these MSU crystal formations appear to indicate that besides the crystals formed in the surface of joint cartilages, the content of toph can drain into the joint fluid, also contributing to the presence of crystals in it; the 